\title{
Compliance beim Tragen einer Entlastungshilfe für Ulkusheilung entscheidend
}

Fragestellung: Das Ziel der Studie war die prospektive Erfassung der Abheilung von plantaren Ulzera, auch in Beziehung zur Tragedauer von abnehmbaren Hilfsmitteln zur Entlastung der Wunde.

Hintergrund: Es gibt mehrere Studien, in denen belegt wurde, dass die fehlende Entlastung von plantaren Ulzera bei Patienten mit diabetischer Polyneuropathie die Abheilung deutlich verzögert. In den meisten dieser Studien wurden dafür Unterschiede in der Abheilung der Ulzera bei Verwendung von abnehmbaren und nicht abnehmbaren Entlastungshilfsmitteln verglichen, z.B. von geschlossenen Gipsen/Casts. Es gibt jedoch kaum Studien zur Tragecompliance von abnehmbaren Hilfsmitteln und zu den Ursachen der geringen Akzeptanz.

Patienten und Methoden: In dieser prospektiven, internationalen Multicenterstudie wurden 79 Patienten mit plantaren Ulzera untersucht, 46 Patienten aus Großbritannien und 33 aus den USA. Die Studiendauer betrug 6 Wochen. Die Ulzera waren überwiegend oberflächlich und nicht ischämisch; University of Texas Classification (1A 72\%; 1B 11\%; 2A 15\%; 2B 1\%). Die meisten Patienten erhielten eine abnehmbare Orthese

Originalie

Crews RT, Shen BJ, Campbell, et al. Role and Determinants of Adherence to Off-loading in Diabetic Foot Ulcer Healing. A Prospective Investigation. Diabetes Care 2016, 39:1371-7. (77\%), die anderen überwiegend Therapieschuhe. Die Compliance bezüglich des Tragens der Hilfsmittel wurde ermittelt durch Aktivitätsmonitore, wobei einer am Gürtel getragen wurde und der andere, ohne Wissen des
Patienten, im Walker eingebaut war. Des Weiteren kamen verschiedene psychologische Fragebogen zur Anwendung: Hospital Anxiety and Depression Scale (HADS), Neuropathy and Foot Ulcer Quality of Life (NeuroQoL) und das Revised Illness Perception Questionnaire (IPQ-R).

Ergebnisse: Die Compliance bezüglich der Entlastung wurde über $35 \pm 10$ Tage erhoben und die Hilfsmittel wurden während $59 \pm 22 \%$ der täglichen Aktivität der Patienten getragen. In der multivariaten Analyse ergab sich, dass eine bessere Abheilung des Ulkus nach 6 Wochen assoziiert war mit einer höheren Compliance bezüglich des Tragens der Hilfsmittel sowie außerdem mit einem kleinen Ulkus bei Studienbeginn und der Behandlung in Großbritannien. Eine hohe Compliance bezüglich des Tragens der Hilfsmittel war signifikant assoziiert mit größeren und schweren Ulzera bei Studienbeginn, einer schwereren Neuropathie und mehr Schmerzen im Fuß. Eine niedrige Compliance war hingegen bei denjenigen Patienten zu erkennen, die entsprechend dem NeuroQoL bei Beginn der Studie eine höhere Instabilität beim Stehen aufwiesen. Die HADS- und IPQ-R-Ergebnisse waren hingegen nicht assoziiert mit der Compliance bezüglich des Tragens der Hilfsmittel zur Entlastung.

Schlussfolgerungen: Eine hohe Compliance bezüglich des Tragens der Entlastungshilfsmittel bewirkte eine bessere Abheilung der plantaren Ulzera. Eine höhere Unsicherheit beim Stehen, wie sie bei älteren Patienten mit diabetischer Neuropathie häufig besteht, war hingegen ein starker Indikator dafür, dass das Hilfsmittel nicht regelmäßig getragen wird. Diese Tatsache sollte bei der Auswahl des Hilfsmittels berücksichtigt werden.

\section{- Kommentar von Prof. Dr. med. Maximilian Spraul}

\section{Entlastungshilfe sollte an Fähigkeiten des Patienten angepasst sein}

Diese sehr interessante Studie zeigt, dass viele Patienten mit plantaren Ulzera ihr verordnetes Hilfsmittel nicht regelmäßig tragen. Eine sorgfältige Beurteilung, ob das verordnete Hilfsmittel es dem Patienten ermöglicht überhaupt noch sicher zu gehen, ist immer notwendig. Diese klinische Beobachtung hat auch dazu geführt, dass sogenannte Vorfußentlastungsschuhe („halbe Schuhe“) mit alleiniger Belastungssohle im Rückfuß kaum noch verwendet werden, da ältere Patienten damit kaum sicher Laufen können. Neben all diesen Faktoren ist aber auch die häufig unzureichende Qualität der Hilfsmittel, insbesondere der diabetesadaptierten Bettungen verantwortlich dafür, dass Ulzera nicht abheilen. Am besten wäre natürlich die Verwendung des Goldstandards zur Therapie von plantaren Ulzera, einem geschlossenen Gips/Cast. Dieser wird in
Deutschland aber kaum verwendet, vor allem da er von den Krankenkassen nicht bezahlt wird.

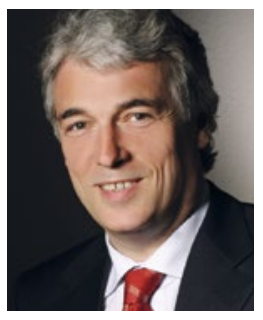

Prof. Dr. med. Maximilian Spraul

Mathias-Spital \& Jakobi-Krankenhaus Interdisziplinäres Diabetes-Fuß-Zentrum Frankenburgstr. 31, 48431 Rheine m.spraul@mathias-spital.de 